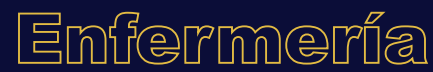

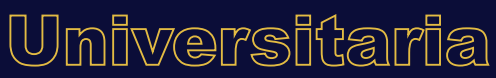

\section{Seguridad del paciente: percepción de estudiantes de una universidad pública}

\author{
Patient safety: Perceptions of \\ students in a public university
}

Segurança do paciente: percepção de estudantes de uma universidade pública

\section{D.E. Hernández-Herrera ${ }^{\text {a1 }}$, A.A. Turrubiates- Piña $^{b_{2}}$, E. Villarreal-Ríos ${ }^{c_{3}}$, E. Vega-Infante ${ }^{d_{1}}$, E.N. Morelos-García $^{\mathrm{e}}, \mathrm{H}$. Castañeda-Hidalgo ${ }^{\mathrm{fi}}{ }^{*}$}

ORCID

a $0000-0001-8728-2570$

do000-0003-1093-2648

b $0000-0001-9206-827 \mathrm{X}$

e $0000-0002-3344-8413$

c $0000-0002-5455-2383$

${ }^{\mathrm{f}} \mathrm{0000-0002-6262-4578}$

${ }^{1}$ Universidad Autónoma de Tamaulipas, Facultad de Enfermería, Tampico, Tamaulipas, México

${ }^{2}$ Instituto Mexicano del Seguro Social, Ciudad Victoria, Tamaulipas, México

3 Instituto Mexicano del Seguro Social, Unidad de Investigación Epidemiológica y de Servicios de Salud, Querétaro, Querétaro, México

Recibido: 06 diciembre 2020

Aceptado: 20 abril 2021

\section{RESUMEN}

Introducción: La seguridad del paciente es una prioridad mundial para los sistemas de salud. Las instituciones educativas formadoras de recursos humanos deben incluir la temática en su currículo.

Objetivo: Identificar la percepción sobre la seguridad del paciente en los estudiantes de licenciatura en enfermería.

\footnotetext{
*Autora para correspondencia. Correo electrónico: hcastane@docentes.uat.edu.mx https://doi.org/10.22201/eneo.23958421e.2021.2.1037

1665-7063/@ 2021 Universidad Nacional Autónoma de México, Escuela Nacional de Enfermería y Obstetricia. Este es un artículo Open Access bajo la licencia CC BY-NC-ND (http://creativecommons.org/licenses/by-nc-nd/4.o/).
} 
Metodología: Estudio descriptivo y transversal. Población total de 516 alumnos de licenciatura en enfermería de la Universidad Autónoma de Tamaulipas, México. Se utilizó el cuestionario APOQS-III y se diseñó una cédula con variables sociodemográficas.

Resultados: La mayoría de los participantes son mujeres, edad promedio: 20 años. La mitad de los alumnos no tiene cursos que avalen sus conocimientos sobre seguridad del paciente. En la dimensión entrenamiento recibido, 63.5 \% concuerda que la formación de pregrado los capacita para entender las causas de un evento adverso por enfermería, los habilita para prevenir errores y desarrollar una adecuada comprensión en materia de seguridad. 50.3\% está totalmente de acuerdo en la dimensión confianza en el reporte del error y es semejante la proporción en la dimensión horas de trabajo como causas de error.

Discusión: La identificación positiva de la percepción que se tiene acerca de la seguridad del paciente por parte de los estudiantes, favorece desde el pregrado la creación de una cultura de seguridad que fomenta la confianza y el interés de estos para desarrollar un enfoque integrado en el tema estudiado.

Conclusiones: Los estudiantes en su mayoría refieren actitudes positivas confiados en que su entrenamiento sobre seguridad los prepara para entender y prevenir las causas de errores en seguridad del paciente.

Palabras claves: Conocimiento; percepción; seguridad del paciente; estudiantes de enfermería; México.

\section{ABSTRACT}

Introduction: Patient safety is a priority of health systems worldwide and therefore, the education institutions responsible for the formation of human resources for health must address this goal in detail.

Objective: To identify the perceptions of baccalaureate nursing students regarding patient safety.

Methodology: This is a descriptive and transversal study on 516 baccalaureates in nursing students from the Universidad Autónoma de Tamaulipas, Mexico. The APOS-III and a specific social-demographic data questionnaire were used.

Results: The majority of the participants were female. The average age was 20 years old. Nearly half of the students do not have enough courses to strengthen their knowledge on patient safety. Regarding the training received, $63.5 \%$ of the participants agree that the baccalaureate level formation prepares them to understand the causes of adverse events in nursing, to prevent errors, and to have an adequate understanding of security. $50.3 \%$ of the participants approved the dimension's confidence approach regarding error reporting. Many participants considered that the excessive long hours working dimension is a cause of errors.

Discussion: The perception of patient safety as being very important favors and further promotes a culture of security.

Conclusions: The majority of the students trust their training regarding patient safety and know how to prevent possible causes of errors.

Keywords: Knowledge; perception; patient safety; students, nursing; Mexico. 


\section{RESUMO}

Introdução: A segurança do paciente é uma prioridade mundial para os sistemas de saúde. As instituições educacionais formadoras de recursos humanos devem incluir a temática em seu currículo.

Objetivo: Identificar a percepção sobre a segurança do paciente nos estudantes de graduação em enfermagem.

Metodologia: Estudo descritivo e transversal. População total de 516 alunos de graduação em enfermagem da Universidad Autónoma de Tamaulipas, México. Foi utilizado o questionário APQS-III e realizou-se um questionário com variáveis sociodemográficas.

Resultados: A maioria dos participantes são mulheres, idade média: 20 anos. Metade dos estudantes não tem cursos que avaliem seus conhecimentos sobre segurança do paciente. Na dimensão do treinamento recebido, 63,5 \% concordam que a formação de graduação os treina para entender as causas de um evento de enfermagem adverso, lhes permite prevenir erros e desenvolver uma compreensão adequada da segurança. 50,3 \% concordam fortemente sobre a dimensão de confiança no relatório dos erros e a proporção e da mesma forma sobre a dimensão das horas de trabalho como causa dos erros.

Discussão: A identificação positiva da percepção que se tem sobre a segurança do paciente por parte dos alunos, favorece desde a graduação a criação de uma cultura de segurança que fomente a confiança e o interesse destes em desenvolver uma abordagem integrada na o assunto estudado.

Conclusões: A maioria dos alunos relata atitudes positivas, confiantes de que seu treinamento em segurança os prepara para compreender e prevenir as causas dos erros na segurança do paciente.

Palavras chave: Conhecimento; percepção; segurança do paciente; estudantes de enfermagem; México.

\section{INTRODUCCIÓN}

La seguridad en la atención de salud representa uno de los temas prioritarios tanto a nivel mundial como nacional. En el año 2004, los estados miembros de la Organización Mundial de la Salud (OMS) formaron la Alianza Mundial para la Seguridad del Paciente, la cual tiene como fin principal impulsar la investigación como elemento esencial para mejorar la seguridad en la atención sanitaria.

A nivel mundial, en las últimas décadas los eventos adversos (EA) que generaron mayor interés investigativo fueron las infecciones asociadas a la atención de salud (IAAS). Actualmente, se reconoce que otros EA incrementan de manera significativa los costos y la morbimortalidad de los pacientes hospitalizados, debido a la aparición de úlceras por presión, caídas de pacientes, errores de medicación, cirugía en paciente equivocado o cirugía en sitio equivocado, identificación incorrecta de pacientes, entre otros; situación que ha producido cambios a nivel político, económico y social, mediante la construcción de nuevas instituciones normativas de carácter sanitario². La Organización Mundial de la Salud define la Seguridad del Paciente (SP) como la reducción de riesgos de daños innecesarios a un mínimo aceptable, según los conocimientos del momento, los recursos disponibles y el contexto, relacionando esta seguridad con la disminución de riesgos asociados a la atención sanitaria, la cual recibe servicios de promoción, mantenimiento, vigilancia o restablecimiento de la salud $^{3}$ 
En el 2017 se estableció en México un acuerdo de obligatoriedad en el Sistema Nacional de Salud que consiste en la implementación de las acciones esenciales para la SP, las cuales quedaron registradas en el Diario Oficial de la Federación`. El Gobierno de la República incorporó la SP en las políticas públicas que se enunciaron en diferentes documentos oficiales como lo son el Plan Nacional de Desarrollo5 y el Programa Sectorial de Salud 2019-2024 ${ }^{6}$. Asimismo, la Comisión Permanente de Enfermería coordinó la construcción de indicadores de calidad con respecto a la administración de medicamentos vía oral, vigilancia y control de accesos venosos instalados y trato digno de enfermería. Si bien estos tres indicadores se relacionan con la SP, actualmente dicha comisión se encuentra trabajando en el desarrollo de otros indicadores de cuidado?.

Sin duda, en los sistemas de salud la conjugación de los avances tecnológicos y la complejidad en la atención médica aportan beneficios, aunque también pueden provocar graves deficiencias en la calidad de la atención y la seguridad del paciente. La alta prevalencia de riesgos clínicos y los incidentes de seguridad han aumentado las preocupaciones y los desafíos en esta área ${ }^{8,9}$.

En ese sentido, la seguridad del paciente y el error humano deben ser abordados desde la formación, dado que el enfermero/a es el profesional de la salud que tiene mayor contacto con el paciente. Durante la práctica, estos últimos son temas importantes y esenciales ${ }^{10}$, además de otros como: calidad de la atención, gestión del cuidado y uso de indicadores de atención para que los estudiantes conozcan los factores asociados a la ocurrencia de los EA e identifiquen cuáles son los recursos mínimos indispensables que se requieren para realizar los diferentes procedimientos al proporcionar atención, de tal forma que se puedan prevenir errores en el cuidado ante la falta de infraestructura ${ }^{11}$.

Dada la importancia indiscutida de la SP, como dimensión clave de la calidad asistencial y su impacto en las políticas sanitarias a nivel mundial, es relevante que los profesionales enfermeros trabajen en perfeccionar la calidad de la formación, la asistencia, la investigación y la gestión del cuidado para lograr dos objetivos comunes: cultura de seguridad y seguridad del paciente ${ }^{12,13}$. La seguridad clínica debe ser un objetivo sustancial de la educación de enfermería en pregrado, donde es fundamental instruir a los futuros profesionales en temas relevantes como la calidad del cuidado, epidemiología de los EA, además de su prevención y control ${ }^{14}$. La calidad, el contenido y la oferta de la educación de enfermería acerca de la SP tendrán un impacto significativo en la cultura de seguridad que practiquen los futuros profesionales de enfermería en sus entornos clínicos. Por ende, las instituciones formadoras deben discutir y ampliar esta temática al medio académico y profesional para llevar a cabo acciones que puedan prevenir la ocurrencia de incidentes durante la prestación del cuidado.

Al respecto, los estudiantes precisan comprender que los errores acontecen, pero para un cuidado más seguro se debe conocer y aprender acerca de la SP. También deben tener presente que los cursos del área de la salud poseen un papel fundamental, pues la asociación de la enseñanza con la práctica posibilita la identificación de análisis de los riesgos en la asistencia, además de que busca estrategias para que haya mejoras en los procesos de trabajo.

Diversos autores han estudiado la SP y aspectos como la percepción de las competencias y actitudes de los estudiantes de licenciatura en enfermería, así como la integración del tema en el currículo desde el pregrado. Los principales hallazgos reportan la actitud adecuada hacia la variable en estudio por parte de los estudiantes, así como el deseo de aprender al respecto; los temas relevantes para el aprendizaje incluyeron los EA, la acción anticipatoria en entornos complejos y la enfermería centrada en la SP ${ }^{13-18}$. Por ello, el objetivo de esta investigación fue identificar la percepción que tienen los estudiantes acerca de la seguridad del paciente para involucrarlos en el aprendizaje significativo de ese fenómeno. 


\section{METODOLOGÍA}

Se realizó un diseño de investigación de tipo descriptivo, cuantitativo. La población encuestada fueron estudiantes inscritos de tercer a octavo semestre en el periodo escolar 2018-1 correspondiente a los meses de enero a junio del 2018 de la Unidad Académica Multidisciplinaria Mante Centro de la Universidad Autónoma de Tamaulipas (UAMMC-UAT), participaron 516 alumnos de un total de 531. Se excluyeron a estudiantes inscritos en los semestres inferiores a tercer semestre porque aún no asisten a prácticas clínicas y aquellos que estaban ausentes del salón de clase al momento de la encuesta.

Se utilizó el instrumento Attitudes to Patient Safety Questionnaire (APOS-III) ${ }^{19}$, el cual es una herramienta útil para identificar el conocimiento, las creencias y las actitudes hacia la seguridad del paciente que tienen los estudiantes de carreras biomédicas. El instrumento está compuesto de 26 ítems divididos en 9 dimensiones: entrenamiento recibido en la seguridad del paciente (1-3), confianza en el reporte del error (4-6), horas de trabajo como causa de error (7-9), inevitabilidad del error (10-12), incompetencia profesional como causa de error (13-16), responsabilidad de la comunicación (17-19), funcionamiento del equipo (2O-21), compromiso del paciente en la reducción del error (22-23), e importancia de la seguridad del paciente en el currículum (24-26). Las respuestas fueron puntuadas en una escala Likert: 1) totalmente en desacuerdo, 2) moderadamente en desacuerdo, 3) ni de acuerdo ni en desacuerdo, 4) moderadamente de acuerdo y 5) totalmente de acuerdo. El instrumento reportó una fiabilidad global de 0.695 mediante Alpha de Cronbach ${ }^{19}$.

Se contó con la aprobación del Comité de Ética en Investigación de la Facultad de Enfermería de Tampico, además del consentimiento informado por escrito para los estudiantes. Para el procesamiento de los datos se usó el paquete estadístico Statistic Package for Social Sciences (SPSS) versión 18. Se utilizó estadística descriptiva por medio de frecuencias, porcentajes e intervalos de confianza para describir las variables de estudio, además de la media aritmética para la variable edad. Para determinar la confiabilidad del instrumento de recolección de datos se calculó el Alpha de Cronbach, el cual fue de .90. Asimismo, se diseñó una cédula con variables sociodemográficas como sexo, edad, semestre y cursos relacionados con la seguridad del paciente.

\section{RESULTADOS}

Participaron 516 alumnos, lo que corresponde al 97.1 \% de la población total de la Licenciatura en Enfermería de la UAMMC-UAT. El promedio de edad fue de 20 años, con una mínima de 18 y máxima de 38. La mayoría de los participantes cursaba el sexto semestre (33.5\%). Poco más de la mitad (53.5\%) de ellos estaba inscrito en turno vespertino. Del total de los alumnos participantes, solo $34.5 \%(f=$ 178) de la población contaba con algún curso relacionado con la SP (Tabla 1).

\begin{tabular}{|c|c|c|}
\hline Variable & $f$ & $\%$ \\
\hline \multicolumn{3}{|l|}{ Sexo } \\
\hline Hombre & 178 & 34.5 \\
\hline Mujer & 338 & 65.5 \\
\hline \multicolumn{3}{|l|}{ Semestre } \\
\hline Tercero & 32 & 6.2 \\
\hline Cuarto & 154 & 29.8 \\
\hline Sexto & 173 & 33.5 \\
\hline Octavo & 157 & 30.5 \\
\hline \multicolumn{3}{|l|}{ Turno } \\
\hline Matutino & 240 & 46.5 \\
\hline Vespertino & 276 & 53.5 \\
\hline \multicolumn{3}{|c|}{ Cursos relacionados con la Seguridad del Paciente } \\
\hline Sí & 178 & 34.5 \\
\hline No & 338 & 65.5 \\
\hline
\end{tabular}


En cuanto a los resultados correspondientes a la percepción de los estudiantes en la dimensión entrenamiento recibido en la seguridad del paciente, $63.5 \%$ (IC $95 \%$; 69.8 - 77.4) de los encuestados estuvo totalmente de acuerdo en que la formación los prepara para entender las causas de error en enfermería, los capacita para tener adecuada comprensión en materia de seguridad y consideran que la formación recibida los habilita para prevenir errores. Por el contrario, una proporción mínima (4.5\% [IC $95 \% ; 2.7$ - 6.3]) refirió estar totalmente en desacuerdo (Tabla 2).

Tabla 2. Percepción de estudiantes de enfermeria sobre la seguridad del paciente

\begin{tabular}{|c|c|c|c|c|c|}
\hline \multirow[t]{2}{*}{ Dimensión } & $\begin{array}{l}\text { Totalmente } \\
\text { en } \\
\text { desacuerdo }\end{array}$ & $\begin{array}{l}\text { Moderadamente } \\
\text { en desacuerdo }\end{array}$ & $\begin{array}{c}\text { Ni de } \\
\text { acuerdo } \\
\text { ni en } \\
\text { desacuerdo }\end{array}$ & $\begin{array}{l}\text { Moderadamente } \\
\text { de acuerdo }\end{array}$ & $\begin{array}{l}\text { Totalmente } \\
\text { de acuerdo }\end{array}$ \\
\hline & \multicolumn{5}{|c|}{ Porcentaje } \\
\hline $\begin{array}{l}\text { 1. Entrenamiento } \\
\text { recibido en seguridad } \\
\text { del paciente }\end{array}$ & $\begin{array}{c}4.5 \\
(2.7-6.3)\end{array}$ & $\begin{array}{c}2.7 \\
(1.3-4.1)\end{array}$ & $\begin{array}{c}4.5 \\
(2.7-6.3)\end{array}$ & $\begin{array}{c}24.8 \\
(21.1-28.5)\end{array}$ & $\begin{array}{c}63.5 \\
(69.8-77.4)\end{array}$ \\
\hline $\begin{array}{l}\text { 2. Confianza en el } \\
\text { reporte del error }\end{array}$ & $\begin{array}{c}3.9 \\
(2.2-5.6)\end{array}$ & $\begin{array}{c}4.7 \\
(2.9-6.5)\end{array}$ & $\begin{array}{c}14.5 \\
(11.5-17.5)\end{array}$ & $\begin{array}{c}26.6 \\
(22.8-30.4)\end{array}$ & $\begin{array}{c}50.3 \\
(46.1-54.7) \\
\end{array}$ \\
\hline $\begin{array}{l}\text { 3. Horas de trabajo } \\
\text { como causa de error }\end{array}$ & $\begin{array}{c}4.7 \\
(2.9-6.5)\end{array}$ & $\begin{array}{c}5.2 \\
(3.3-7.1)\end{array}$ & $\begin{array}{c}17.6 \\
(14.3-20.9)\end{array}$ & $\begin{array}{c}22.7 \\
(19.1-26.3)\end{array}$ & $\begin{array}{c}49.8 \\
(45.1-53.7)\end{array}$ \\
\hline $\begin{array}{l}\text { 4. Inevitabilidad del } \\
\text { error }\end{array}$ & $\begin{array}{c}7.4 \\
(5.1-9.7) \\
\end{array}$ & $\begin{array}{c}5.0 \\
3.1-6.9\end{array}$ & $\begin{array}{c}20.2 \\
(16.7-23.7)\end{array}$ & $\begin{array}{c}38.8 \\
(34.6-43.0)\end{array}$ & $\begin{array}{c}28.6 \\
(24.8-32.6)\end{array}$ \\
\hline $\begin{array}{l}\text { 5. Incompetencia } \\
\text { profesional como } \\
\text { causa de error }\end{array}$ & $\begin{array}{c}2.7 \\
(1.3-4.1)\end{array}$ & $\begin{array}{c}4.1 \\
(2.4-5.8)\end{array}$ & $\begin{array}{c}14.5 \\
(11.5-17.5)\end{array}$ & $\begin{array}{c}33.4 \\
(29.0-37.2)\end{array}$ & $\begin{array}{c}45.3 \\
(41.0-49.6)\end{array}$ \\
\hline $\begin{array}{l}\text { 6. Responsabilidad de } \\
\text { la comunicación }\end{array}$ & $\begin{array}{c}2.3 \\
(1.0-3.6) \\
\end{array}$ & $\begin{array}{c}7.4 \\
(5.1-9.7) \\
\end{array}$ & $\begin{array}{c}20.2 \\
(16.7-23.7)\end{array}$ & $\begin{array}{c}31.0 \\
(27.0-35.0)\end{array}$ & $\begin{array}{c}39.1 \\
(34.9-43.3)\end{array}$ \\
\hline $\begin{array}{l}\text { 7. Funcionamiento del } \\
\text { equipo }\end{array}$ & $\begin{array}{c}4.3 \\
(2.5-6.1)\end{array}$ & $\begin{array}{c}1.4 \\
(0.4-2.4)\end{array}$ & $\begin{array}{c}2.3 \\
(1.0-3.6)\end{array}$ & $\begin{array}{c}24.8 \\
(21.1-28.5)\end{array}$ & $\begin{array}{c}67.2 \\
(63.1-71.3)\end{array}$ \\
\hline $\begin{array}{l}\text { 8. Compromiso del } \\
\text { paciente en la } \\
\text { reducción del error }\end{array}$ & $\begin{array}{c}2.9 \\
(1.5-4.3)\end{array}$ & $\begin{array}{c}3.1 \\
(1.6-4.6)\end{array}$ & $\begin{array}{c}6.0 \\
(4.0-8.0)\end{array}$ & $\begin{array}{c}34.3 \\
(30.2-38.4)\end{array}$ & $\begin{array}{c}53.7 \\
(49.4-58.0)\end{array}$ \\
\hline $\begin{array}{l}\text { 9. Importancia de la } \\
\text { seguridad del paciente } \\
\text { en el currículum }\end{array}$ & $\begin{array}{c}3.3 \\
(1.8-4.8)\end{array}$ & $\begin{array}{c}1.7 \\
(0.6-2.8)\end{array}$ & $\begin{array}{c}7.2 \\
(5.0-9.4)\end{array}$ & $\begin{array}{c}35.3 \\
(31.2-39.4)\end{array}$ & $\begin{array}{c}52.5 \\
(48.2-56.8)\end{array}$ \\
\hline
\end{tabular}

Nota: Los valores entre paréntesis corresponden al intervalo de confianza 95\%. n=516

Dentro de la dimensión confianza en el reporte del error; que incluye el reporte de los errores cometidos por sí mismos u otras personas del equipo de salud y si considera que podría sentirse cómodo al momento de externar abiertamente a la supervisora algún error cometido, que resultó en un daño potencial o real del paciente; $50.3 \%$ (IC $95 \%$; 46.1 - 54.7) de los estudiantes refirió estar totalmente de acuerdo y 26.6 \% (IC $95 \%$; 22.8 - 30.4) moderadamente de acuerdo. En la dimensión horas de trabajo como causa de error $49.8 \%$ (IC $95 \%$; 45.1 - 53.7) de la población encuestada refiere que los turnos de trabajo más cortos para el personal de enfermería permitirá reducir los errores al momento de brindar atención al paciente, argumentan que el no tomar descansos regulares durante 
los turnos se aumenta el riesgo de cometer errores; el 4.7 \% (IC $95 \%$; 2.9 - 6.5) están totalmente en desacuerdo en que los horarios de trabajo influyan en el aumento de errores (Tabla 2).

Para la dimensión inevitabilidad del error, 38.8 \% (IC 95 \%; 34.6 - 43.0) de la población encuestada está moderadamente de acuerdo con que el error humano es inevitable e incluso el personal de enfermería más competente o experto comete errores, mientras que 5 \% (IC 95 \%; 3.1 - 6.9) estuvo moderadamente en desacuerdo y creen que un verdadero profesional no comete errores (Tabla 2). En la dimensión incompetencia profesional como causa de error 45.3 \% (IC 95\%; 41.0 - 49.6) se encuentra totalmente de acuerdo en que los errores en enfermería derivan de profesionales descuidados, pues si estos prestan más atención en el trabajo los errores en enfermería serían evitados. Por su parte, $2.7 \%$ (IC $95 \% ; 1.3$ - 4.1) se encuentra totalmente en desacuerdo con que los errores sean secundarios a lo antes mencionado (Tabla 2).

Sobre la dimensión responsabilidad de la comunicación, 39.1 \% (IC 95 \%; 34.9 - 43.3) de los estudiantes encuestados refirió estar totalmente de acuerdo que es necesario reportar todos los errores, sobre todo los que podrían derivar en eventos adversos para el paciente, el personal de enfermería tiene la responsabilidad de comunicar el error al paciente sólo si este se ve afectado; el $2.3 \%$ (IC 95 \%; 1.0 - 3.6) refirió estar totalmente en desacuerdo sobre comunicar los errores (Tabla 2).

Con respecto a la dimensión funcionamiento del equipo, el análisis de resultados mostró que un mejor trabajo en equipo multidisciplinario reducirá los errores en enfermería, así como enseñar habilidades de trabajo en equipo reducirá los errores. Por ello, destaca la importancia del trabajo en equipo para reducción de errores, pues 67.2 \% (IC 95 \%; 63.1 - 71.3) se encontró totalmente de acuerdo (Tabla 2).

En relación con la dimensión compromiso del paciente en la reducción del error, la cual hace referencia al rol del paciente sobre su seguridad, 53.7 \% (IC $95 \%$; 49.4 - 58.0) de los estudiantes encuestados se encontró totalmente de acuerdo y 34.3 \% (IC 95 \%; 30.2 - 38.4) moderadamente de acuerdo con que los pacientes tienen un rol importante en la reducción del error en enfermería y estimularlos a estar más involucrados en su cuidado puede ayudar a reducir el riesgo de ocurrencia de errores en enfermería (Tabla 2).

Para la dimensión importancia de la seguridad del paciente en el currículum, $52.5 \%$ (IC $95 \%$; 48.2 - 56.8) de los encuestados se mostró totalmente de acuerdo y $35.3 \%$ (IC 95 \%; 31.2 - 39.4) moderadamente de acuerdo en que la formación en SP se debe considerar una prioridad para convertirse en un profesional de enfermería eficiente (Tabla 2).

\section{DISCUSIÓN}

El presente estudio contribuye a identificar la percepción de los estudiantes de pregrado en enfermería sobre la SP, pues al ser una parte activa del proceso de atención requieren de una mayor integración entre el aprendizaje teórico y el clínico ${ }^{13}$. Aunado a esto, se debe tener en cuenta que los estudiantes presentan actitudes positivas cuando se exponen a la realidad de la práctica clínica ${ }^{20}$ durante su formación y al incorporarse a las instituciones deben integrarse a la cultura para evitar los EA.

Cuando se analiza la dimensión entrenamiento recibido se aprecia que este tipo de aprendizaje es percibido por el estudiante en términos adecuados, lo que indica la capacitación en la escuela o el entrenamiento hospitalario que ha recibido en la institución de salud como parte de sus prácticas clínicas, reconociendo así al proceso de formación como el principal responsable en la institución de una cultura de seguridad positiva ${ }^{21}$. 
Esta misma actitud se observa en la dimensión confianza en el reporte de error, la cual es similar a los resultados obtenidos en otro estudio ${ }^{22}$ en el que la mayoría de los estudiantes declararon comunicar siempre la ocurrencia de errores. Haciendo una proyección a futuro, esto último será la evidencia de un personal de salud responsable en el establecimiento de sistemas para evitar errores, condición que puede indicar un alto grado de responsabilidad por parte del estudiante de enfermería.

Ahora bien, los estudiantes identifican el exceso de trabajo como un factor de riesgo que favorece el cometer errores y con ello el incremento en la posibilidad de los EA. En dicho sentido, las horas de trabajo como causa de error son sin duda un aspecto importante, pues el personal de enfermería son los profesionales de salud que están más expuestos a cometerlos porque son los que pasan mayor tiempo con los pacientes 23 .

Si bien es cierto que el estudiante reconoce la importancia de su actividad para evitar los EA, también está consciente que existen condiciones del medio ambiente y del paciente, los cuales favorecen su presencia; por ello debe incorporar a la institución y al paciente como sujetos activos en la prevención de EA. A través de la cultura de prevención se le da valor al paciente como protagonista y conocedor de su propio cuidado ${ }^{10}$, además de asignarle un rol activo, esto le permitirá alejarlo del rol pasivo que tradicionalmente se le había concedido en su cuidado, generando así un cambio en la cultura de prevención de los EA y de la SP.

Lo antes descrito nos permite identificar la importancia del rol del personal de enfermería para evitar los EA; sin embargo, la incompetencia profesional es una de las causantes de los EA. Los estudiantes pueden no tener internalizado el concepto de seguridad del paciente, pero sí las acciones de cuidado necesarias como no hacer daño ${ }^{24}$. Además, cuando realizan su práctica clínica con personal experimentado se espera que trabajen en equipo y se comuniquen con sus compañeros, que sean cautelosos, pregunten a las enfermeras/os en casos de duda y notifiquen si cometen un error ${ }^{20}$.

Por último, el estudiante de enfermería considera que la SP es un tema que debe formar parte de los planes de estudio. No se sabe si esta perspectiva se adquirió en la escuela o en las prácticas clínicas que los estudiantes de enfermería realizan en los centros hospitalarios desde el $3 .{ }^{\text {er }}$ semestre de la carrera; durante este periodo el estudiante tiene el primer contacto clínico con el paciente, infraestructura y personal del equipo de salud $\mathrm{d}^{24}$. Si bien durante las prácticas el alumno observa que la cultura de SP no es una misión fácil, también lo identifica como un elemento fundamental para desarrollar competencias en la formación del recurso humano en salud, atendiendo de una manera proactiva la prevención de eventos adversos 25 .

\section{CONCLUSIONES}

Se concluye que las percepciones sobre SP de los estudiantes de la licenciatura de enfermería son proactivas y forman parte de su cultura profesional y de atención en el servicio asumiendo la responsabilidad que les corresponde. Sin embargo, esta investigación puede continuarse a través de una intervención educativa en estudiantes que incluya estrategias como estudios de caso o simulación clínica, a fin de contribuir al desarrollo del pensamiento crítico del estudiante y a una actitud positiva. Finalmente, destaca la importancia de formar a los futuros profesionales en materia de seguridad del paciente ajustando los planes de estudio a los aspectos relacionados a este tema.

RESPONSABILIDADES ÉTICAS

Protección de personas y animales. Los autores declaran que no se han realizado experimentos en personas ni animales. 
Confidencialidad. Los autores declaran que se han seguido los protocolos necesarios para la protección de los datos de los informantes.

Derecho a la privacidad y consentimiento informado. Los autores declaran que en este artículo no aparecen datos sobre los informantes.

Conflicto de intereses. Los autores declaran no tener conflictos de intereses.

Financiamiento. Ninguno.

\section{REFERENCIAS}

1. Organización Mundial de la Salud. Alianza mundial para la seguridad del paciente. La investigación en seguridad del paciente. Mayor conocimiento para una atención más segura. Ginebra: OMS; 2008. https://bit.ly/3vA8Oko

2. Aranaz-Andrés JM, Aibar-Remón C, Limón-Ramírez R, Amarilla A, Restrepo FR, Urroz O, et al. Prevalence of adverse events in the hospitals of five Latin America countries: Results of the "Iberoamerican study of adverse events" (IBEAS). BMJ Qual Saf. 2011; 20(12): 1043-51.

https://doi.org/10.1136/bmjqs.2011.051284

3. Organización Mundial de la Salud. Más que palabras. Marco Conceptual de la Clasificación Internacional para la Seguridad del Paciente. Versión 1.1. Informe técnico definitivo. Ginebra: OMS; 2009. http://bit.ly/2IpE7qT

4. Secretaría de Gobernación. Acuerdo por el que se declara la obligatoriedad de la implementación, para todos los integrantes del Sistema Nacional de Salud, del documento denominado Acciones Esenciales para la Seguridad del Paciente. CDMX: SEGOB; 2017. https://bit.ly/3AXSKdy

5. Gobierno de México. Plan Nacional de Desarrollo 2019-2024. México: Gobierno de México; s/f. https://bit.ly/3C5J6aa

6. Secretaría de Salud. Programa Sectorial de Salud 2019-2024. México: SSA; s/f. https://bit.ly/3pmsFTi

7. Comisión Interinstitucional de Enfermería. Evaluación de la calidad de los servicios de enfermería. Tres indicadores de aplicación hospitalaria. México: SSA/DGCES/CIE; 2003. https://bit.ly/2Z6KkTU

8. Farokhzadian J, Dehghan-Nayeri N, Borhani F. Assessment of clinical risk management system in hospitals: An approach for quality improvement. Glob J Health Sci. 2015; 7(5): 294-303.

https://doi.org/10.5539/gjhs.v7n5p294

9. Farokhzadian J, Dehghan-Nayeri N, Borhani F. Rocky milieu: Challenges of effective integration of clinical risk management into hospitals in Iran. Int J Qualitative Stud Health Well-being. 2015; 10: 1-11. https://doi.org/10.3402/qhw.v10.27040

10. Figuereido-Rodrigues G, Salgado-Castro TC, Ferreira-Vitorio AM. Segurança do paciente: conhecimento e atitudes de enfermeiros em formação. Rev. Recien. 2018: 8(24): 3-14.

https://doi.org/10.24276/rrecien2358-3088.2018.8.24.3-14

11. Zarza-Arizmendi MD, Alba-Leonel A, Salcedo-Álvarez RA. El currículum de enfermería y la seguridad del paciente. Rev. Conamed. 2008; 13: 33-7. https://bit.ly/30P8OSx

12. León-Román C. La seguridad del paciente, una responsabilidad en el acto del cuidado. Rev. cuba. enferm. 2006; 22(3): 1-7. https://bit.ly/3AXOAdW

13. Bianchi M, Bressan V, Cadorin L, Pagnucci N, Tolotti A, Valcarenghi D, et al. Patient safety competencies in undergraduate nursing students: A rapid evidence assessment. J Adv Nurs. 2016; 72(12): 2966-97. https://doi.org/10.1111/jan.13033 
14. Tella S, Liukka M, Jamookeeah D, Smith NJ, Partanen P, Turunen H. What do nursing students learn about patient safety? An integrative literature review. J Nurs Educ. 2014; 53(1): 7-13.

https://doi.org/10.3928/01484834-20131209-04

15. Lee NJ, Jang H, Park SY. Patient safety education and baccalaureate nursing students' patient safety competency: A cross-sectional study. Nurs Health Sci. 2016; 18(2): 163-71.

https://doi.org/10.1111/nhs.12237

16. Stevanin S, Bressan V, Bulfone G, Zanini A, Dante A, Palese A. Knowledge and competence with patient safety as perceived by nursing students: The findings of a cross-sectional study. Nurse Educ Today. 2015; 35(8): 926-34. https://doi.org/10.1016/j.nedt.2015.04.002

17. Mansour M. Factor analysis of nursing students' perception of patient safety education. Nurse Educ Today. 2015; 35(1): 32-7. https://doi.org/10.1016/j.nedt.2014.04.020

18. DeBourgh GA, Prion SK. Patient safety manifesto: A professional imperative for prelicensure nursing education. J Prof Nurs. 2012; 28(2): 110-8. https://doi.org/10.1016/j.profnurs.2011.05.001

19. García-Elorrio E, Macchiavello D, Rodríguez V, Catalano Y, Corna G, Dahinten J, et al. Reporte de conocimientos, creencias y actitudes en calidad de atención y seguridad del paciente en alumnos de pregrado: validando el cuestionario APSO-III modificado. Medwave. 2016; 16(11): 1-6.

https://doi.org/10.5867/medwave.2016.11.6809

20. Cantero-López N, González-Chordá VM, Valero Chillerón MJ, Mena-Tudela D, Andreu-Pejó L, Vila-Candel R, et al. Attitudes of undergraduate nursing students towards patient safety: A quasi-experimetal study. Int. J. Environ. Res. Public Health. 2021; 18: 1-11.

https://doi.org/10.3390/ijerph18041429

21. Ilha P, Radünz V, Vieira-Tourinho FS, Mendes-Marinho M. Segurança do paciente na percepção de acadêmicos de enfermagem. Cogitare Enferm. 2016; 21(esp): 1-10.

https://doi.org/10.5380/ce.v21i5.43620

22. Yoshikawa JM, Catin-de Sousa BE, Sorgini-Peterlini MA, Miyuki-Kusahara D, Gonçalves-Pedreira ML, Ferreira-Machado Avelar A. Comprehension of undergraduate students in nursing and medicine on patient safety. Acta paul. enferm. 2013; 26(1): 21-9.

https://doi.org/10.1590/S0103-21002013000100005

23. De Costa-Machado Duarte S, Azevedo-Queiroz AB, Büscher A, Andrade-Conceição Stipp M. El error humano en el cotidiano de la atención de enfermería en terapia intensiva. Rev. Latino-Am. Enfermagem. 2015; 23(6): 1074-81. https://doi.org/10.1590/0104-1169.0479.2651

24. Chitolina-Eberle C, Scheffer-Schell da Silva AP. Compreensão de estudantes de enfermagem sobre a segurança do paciente. Rev. baiana enferm. 2016; 30(4): 1-9. https://bit.ly/31PBM5r

25. Meléndez-Mogollón IC, Macías-Maroto M, Álvarez-González AR. Cultura de seguridad del paciente en la formación de enfermería. Rev. cuba. enferm. 2020; 36(2): 1-14. https://bit.ly/3jGtumn 